Supplementary material for:

\title{
Experimental bottom trawling finds resilience in large-bodied infauna but vulnerability for epifauna and juveniles in the Frisian Front
}

Justin C. Tiano ${ }^{1,3^{*}}$, Karin J. van der Reijden ${ }^{2}$, Sarah O’Flynn ${ }^{1}$, Olivier Beauchard ${ }^{1}$, Sietse van der Ree ${ }^{2}$, Jelmer van der Wees ${ }^{2}$, Tom Ysebaert ${ }^{1,4}$, Karline Soetaert ${ }^{1,3}$

${ }^{1}$ Royal Netherlands Institute for Sea Research (NIOZ) and Utrecht University, Department of Estuarine and Delta Systems, PO box 140, 4400 AC, Yerseke, The Netherlands

${ }^{2}$ Conservation Ecology Group, Groningen Institute for Evolutionary Life Sciences, University of Groningen, P.O. Box 11103, 9700 CC Groningen, The Netherlands.

${ }^{3}$ Ghent University, Department of Biology, Marine Biology Section, Krijgslaan 281/S8, 9000 Ghent, Belgium

${ }^{4}$ Wageningen Marine Research, Wageningen University and Research, PO Box 77, 4400 AB, Yerseke, The Netherlands

*Contact author: Justin.Tiano@nioz.nl 


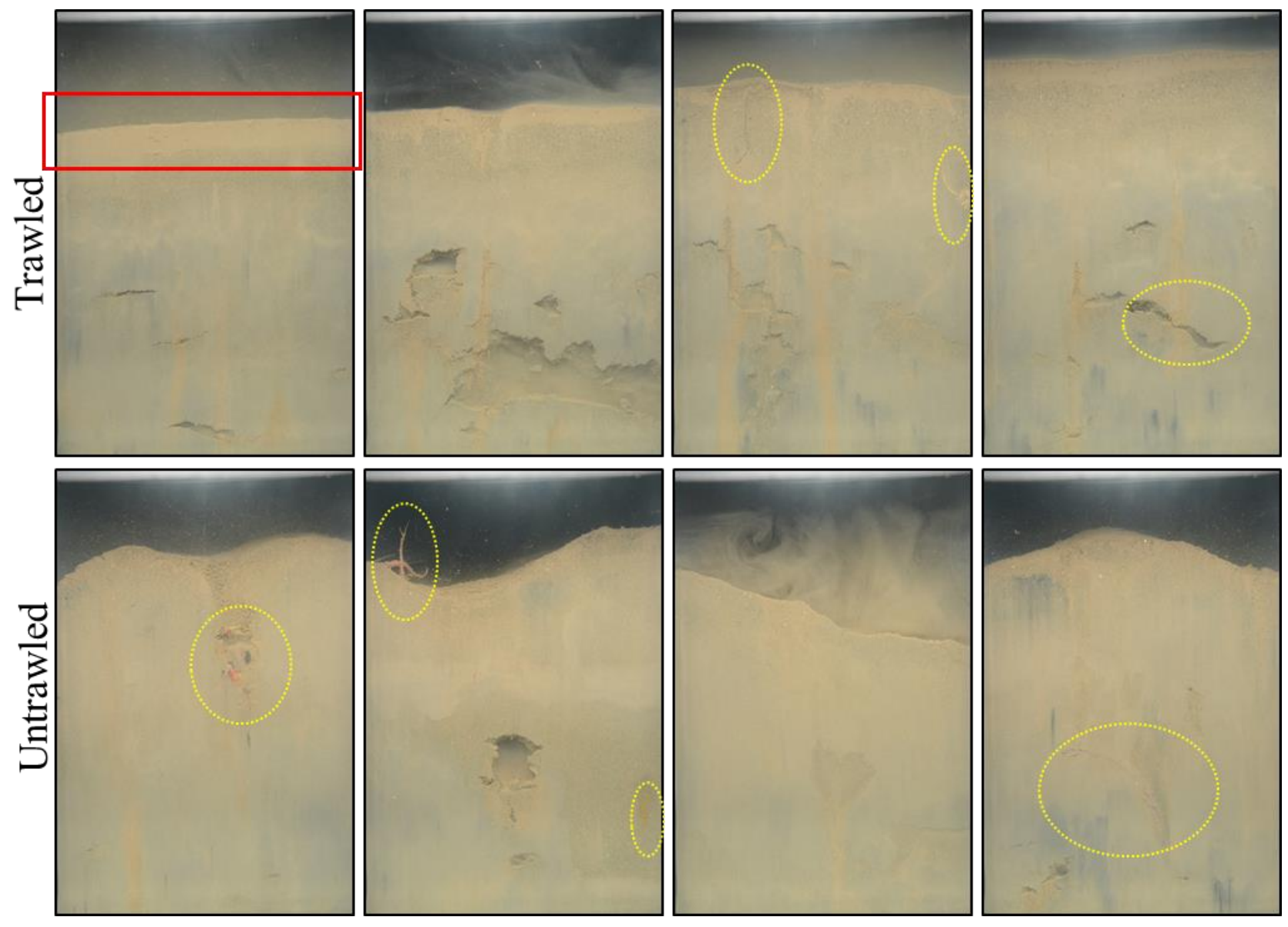

Fig. S1. An assortment of sediment profile images (SPI) displaying beam trawled sediment (top) compared with images from an untrawled reference area (bottom). The red box highlights the characteristic fine sediment layer found in beam trawled images. Infauna from the images are circled in yellow. 
Table S1

Biological traits and modalities

\begin{tabular}{cc}
\hline Biological Trait & Modality \\
\hline \multirow{3}{*}{ Burrowing Depth } & $0 \mathrm{~cm}$ \\
& $5-5 \mathrm{~cm}$ \\
& $>15 \mathrm{~cm}$ \\
\hline \multirow{3}{*}{ Burrowing Depth } & $<1 \mathrm{~cm}$ \\
& $1-3 \mathrm{~cm}$ \\
& $3-10 \mathrm{~cm}$ \\
Body Mass & $10-20 \mathrm{~cm}$ \\
& $>20 \mathrm{~cm}$ \\
\hline \multirow{2}{*}{ Fragility } & Very small \\
& Small \\
& Intermediate \\
& Large \\
& Very Large \\
\hline Motility & Fragile \\
& Intermediate \\
& Robust \\
\hline & Sessile \\
& Tuberculous \\
& Crawler \\
& Crawler - Swimmer \\
\hline
\end{tabular}


Table S2

Trawled vs. untrawled comparisons of epibenthos density (individuals $\mathrm{m}^{-2}$ ), burrow hole density (holes $\mathrm{m}^{-2}$ ), burrow hole size $\left(\mathrm{cm}^{2}\right)$, and burrow hole coverage (\%) from video transects covering beam trawled sediments $(27 \mathrm{~h}$ and $50 \mathrm{~h}$ after disturbance).

\begin{tabular}{|c|c|c|c|c|c|c|}
\hline Parameter & Time step & Disturbance & Observer 1 & Observer 2 & $n$ & p-value \\
\hline \multirow{4}{*}{ 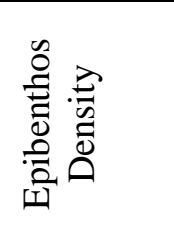 } & \multirow{2}{*}{$\sim 27 \mathrm{~h}$} & Trawled & $0.70 \pm 1.79$ & $0.51 \pm 0.9$ & 41 & \multirow{2}{*}{$<0.0001$} \\
\hline & & Untrawled & $2.28 \pm 4.42$ & $2.46 \pm 5.1$ & 288 & \\
\hline & \multirow{2}{*}{$\sim 50 \mathrm{~h}$} & Trawled & $1.91 \pm 2.95$ & $1.59 \pm 3.2$ & 293 & \multirow[b]{2}{*}{$<0.0001$} \\
\hline & & Untrawled & $3.80 \pm 6.44$ & $2.19 \pm 4.2$ & 95 & \\
\hline \multirow{4}{*}{$\begin{array}{l}\frac{\overparen{D}}{0} \\
\stackrel{0}{0} \\
\stackrel{0}{0}\end{array}$} & \multirow{2}{*}{$\sim 27 \mathrm{~h}$} & Trawled & $112.2 \pm 36.3$ & $62.6 \pm 27.6$ & 41 & \multirow{2}{*}{$<0.0001$} \\
\hline & & Untrawled & $112.3 \pm 40.1$ & $80.4 \pm 31.3$ & 288 & \\
\hline & \multirow{2}{*}{$\sim 50 \mathrm{~h}$} & Trawled & $128.8 \pm 37.8$ & $109.2 \pm 52.6$ & 293 & \multirow{2}{*}{0.68} \\
\hline & & Untrawled & $131.0 \pm 31.6$ & $133.2 \pm 202.4$ & 95 & \\
\hline \multirow{4}{*}{$\begin{array}{l}0 \\
\text { in } \\
0 \\
0 \\
0\end{array}$} & \multirow{2}{*}{$\sim 27 \mathrm{~h}$} & Trawled & $0.0006 \pm 0.0003$ & $0.0006 \pm 0.0003$ & 41 & \multirow{2}{*}{0.002} \\
\hline & & Untrawled & $0.0009 \pm 0.0004$ & $0.0007 \pm 0.0004$ & 288 & \\
\hline & \multirow{2}{*}{$\sim 50 \mathrm{~h}$} & Trawled & $0.0008 \pm 0.0004$ & $0.0008 \pm 0.0004$ & 293 & \multirow{2}{*}{0.67} \\
\hline & & Untrawled & $0.0005 \pm 0.0004$ & $0.0006 \pm 0.0003$ & 95 & \\
\hline \multirow{4}{*}{ 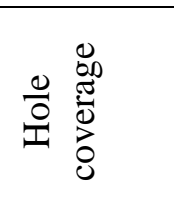 } & \multirow{2}{*}{$\sim 27 \mathrm{~h}$} & Trawled & $0.07 \pm 0.03$ & $0.04 \pm 0.02$ & 41 & \multirow{2}{*}{$<0.0001$} \\
\hline & & Untrawled & $0.10 \pm 0.04$ & $0.05 \pm 0.04$ & 288 & \\
\hline & \multirow{2}{*}{$\sim 50 \mathrm{~h}$} & Trawled & $0.10 \pm 0.04$ & $0.05 \pm 0.04$ & 293 & \multirow{2}{*}{0.005} \\
\hline & & Untrawled & $0.10 \pm 0.04$ & $0.08 \pm 0.12$ & 95 & \\
\hline
\end{tabular}

Table S3

Mean individual densities of juvenile Abra alba and Ophiuroidea per sample per $\mathrm{m}^{2}$

\begin{tabular}{ccc}
\hline \multirow{2}{*}{ Station } & \multicolumn{2}{c}{ Shallow cores } \\
\cline { 2 - 3 } & Abra juv. & Ophiuroidea juv. \\
\hline P0 & 3683.0 & 1126.6 \\
P1 & 993.5 & 241.4 \\
T0 & 2161.1 & 1462.4 \\
T1 & 942.4 & 357.5 \\
T2 & 844.9 & 649.9 \\
T3 & 0 & 433.3 \\
\hline \multicolumn{3}{c}{ Deep cores } \\
Station & \multicolumn{2}{c}{ Ophiuroidea juv. } \\
\cline { 2 - 3 } P0 & Abra juv. & 382.2 \\
P1 & 2700.6 & 127.4 \\
Ref & 396.3 & 509.5 \\
T1 & 2843.3 & 990.8 \\
\hline
\end{tabular}


Table S4.

Top five highest and lowest ordination scores for taxa for Axis 1 (x-axis) and Axis 2 (y-axis) from the Between-Class Analysis (BCA) from shallow subcore and deep boxcores. Scores correspond to the location of the different treatments (T0, T1, T2, T3, P0, P1, Ref) in the BCA ordination plots.

\section{Shallow cores}

\begin{tabular}{lclc}
\hline Taxa with lowest ordination scores & Axis $\mathbf{1}$ & Taxa with highest ordination scores & Axis 1 \\
\hline Abra alba juveniles* & -0.58 & Callianassa subterranea & 0.15 \\
Ophiuroidea juveniles* & -0.35 & Gyge branchialis & 0.06 \\
Amphiura filiformis & -0.27 & Phoronida & 0.05 \\
Spatangoida juveniles* & -0.26 & Bopyroidea & 0.04 \\
Lumbrineris cingulata & -0.25 & Ione thoracica & 0.04 \\
\hline Taxa with lowest ordination scores & Axis $\mathbf{2}$ & Taxa with highest ordination scores & Axis 2 \\
\hline Amphiura filiformis & -0.31 & Spatangoida juveniles* & 0.47 \\
Lumbrineris cingulata & -0.25 & Ophiuroidea juveniles* & 0.43 \\
Nucula nitidosa & -0.21 & Phoronida & 0.29 \\
Oxydromus flexuosus & -0.18 & Tellimya ferruginosa & 0.13 \\
Pholoe baltica & -0.16 & Corystes cassivelaunus juveniles* & 0.12 \\
\hline
\end{tabular}

\section{Deep cores}

\begin{tabular}{lclc}
\hline Taxa with lowest ordination scores & Axis $\mathbf{1}$ & Taxa with highest ordination scores & Axis 1 \\
\hline Abra alba juveniles* & -0.37 & Mediomastus fragilis & 0.35 \\
Diplocirrus glaucus & -0.37 & Atherospio & 0.21 \\
Ophiuroidea juveniles* & -0.33 & Parexogone hebes & 0.16 \\
Eudorella truncatula & -0.257 & Nucula nitidosa & 0.13 \\
Echinoida juveniles* & -0.25 & Prionospio & 0.13 \\
\hline Taxa with lowest ordination scores & $\mathbf{A x i s} \mathbf{2}$ & Taxa with highest ordination scores & Axis 2 \\
\hline Prionospio & -0.27 & Scalibregma inflatum & 0.36 \\
Phaxas pellucidus & -0.22 & Diplocirrus glaucus & 0.26 \\
Podarkeopsis capensis & -0.18 & Abra alba juveniles* & 0.22 \\
Eudorella truncatula & -0.17 & Nemertea & 0.21 \\
Aphroditidae & -0.15 & Polynoidae juveniles* & 0.21 \\
\hline * indicates juvenile taxa & & &
\end{tabular}


Table S5. (1 of 11)

Macrofauna individual densities found in shallow cores.

\begin{tabular}{|c|c|c|c|c|c|c|c|c|}
\hline Station & $\begin{array}{l}\text { Abra } \\
\text { juv. }\end{array}$ & $\begin{array}{l}\text { Abra } \\
\text { alba }\end{array}$ & $\begin{array}{l}\text { Abra } \\
\text { nitida }\end{array}$ & $\begin{array}{c}\text { Abyssoninoe } \\
\text { hibernica }\end{array}$ & $\begin{array}{l}\text { Actinopterygii } \\
(e g g s)\end{array}$ & $\begin{array}{l}\text { Ampelisca } \\
\text { brevicornis }\end{array}$ & $\begin{array}{l}\text { Amphiura } \\
\text { filiformis }\end{array}$ & $\begin{array}{c}\text { Aphroditidae } \\
\text { juv. }\end{array}$ \\
\hline P0 & 31 & 2 & 0 & 0 & 0 & 0 & 5 & 0 \\
\hline P0 & 12 & 0 & 0 & 1 & 0 & 0 & 4 & 0 \\
\hline P0 & 127 & 7 & 0 & 1 & 0 & 0 & 9 & 0 \\
\hline $\mathrm{P} 1$ & 11 & 2 & 0 & 0 & 0 & 0 & 14 & 1 \\
\hline $\mathrm{P} 1$ & 16 & 1 & 0 & 0 & 0 & 0 & 4 & 0 \\
\hline $\mathrm{P} 1$ & 7 & 2 & 0 & 1 & 0 & 0 & 5 & 0 \\
\hline P1 & 24 & 1 & 0 & 0 & 0 & 0 & 13 & 0 \\
\hline $\mathrm{P} 1$ & 21 & 0 & 0 & 1 & 0 & 1 & 7 & 0 \\
\hline $\mathrm{P} 1$ & 9 & 1 & 0 & 2 & 1 & 0 & 7 & 0 \\
\hline $\mathrm{P} 1$ & 19 & 0 & 0 & 0 & 0 & 0 & 5 & 0 \\
\hline T0 & 64 & 1 & 0 & 0 & 2 & 0 & 3 & 0 \\
\hline T0 & 42 & 0 & 0 & 0 & 0 & 0 & 19 & 0 \\
\hline T0 & 9 & 1 & 0 & 0 & 0 & 0 & 18 & 0 \\
\hline T0 & 18 & 1 & 0 & 1 & 0 & 0 & 4 & 1 \\
\hline $\mathrm{T} 1$ & 14 & 0 & 1 & 0 & 0 & 0 & 22 & 0 \\
\hline $\mathrm{T} 1$ & 19 & 1 & 0 & 0 & 2 & 0 & 12 & 0 \\
\hline $\mathrm{T} 1$ & 15 & 1 & 0 & 2 & 0 & 0 & 1 & 1 \\
\hline $\mathrm{T} 1$ & 10 & 0 & 0 & 0 & 0 & 0 & 0 & 0 \\
\hline $\mathrm{T} 2$ & 14 & 0 & 0 & 0 & 0 & 0 & 5 & 0 \\
\hline $\mathrm{T} 2$ & 12 & 0 & 0 & 0 & 1 & 0 & 0 & 0 \\
\hline T3 & 0 & 0 & 0 & 0 & 0 & 0 & 6 & 0 \\
\hline T3 & 0 & 0 & 0 & 0 & 0 & 0 & 0 & 0 \\
\hline $\mathrm{T} 3$ & 0 & 0 & 0 & 0 & 0 & 0 & 0 & 0 \\
\hline
\end{tabular}

Table S5. continued ( 2 of 11$)$

\begin{tabular}{|c|c|c|c|c|c|c|c|}
\hline Station & $\begin{array}{c}\text { Argissa } \\
\text { hamatipes }\end{array}$ & $\begin{array}{c}\text { Atherospio } \\
\text { guillei }\end{array}$ & Bopyroidea & $\begin{array}{l}\text { Callianassa } \\
\text { subterranea }\end{array}$ & $\begin{array}{c}\text { Chamelea } \\
\text { striatula } \\
\text { juv. } \\
\end{array}$ & $\begin{array}{l}\text { Corbula } \\
\text { gibba }\end{array}$ & $\begin{array}{c}\text { Corystes } \\
\text { cassivelaunus } \\
\text { juv. }\end{array}$ \\
\hline P0 & 0 & 0 & 0 & 0 & 0 & 2 & 0 \\
\hline P0 & 0 & 0 & 0 & 0 & 0 & 2 & 0 \\
\hline P0 & 2 & 0 & 0 & 0 & 0 & 1 & 0 \\
\hline $\mathrm{P} 1$ & 0 & 0 & 0 & 0 & 0 & 1 & 0 \\
\hline $\mathrm{P} 1$ & 0 & 0 & 0 & 0 & 0 & 4 & 0 \\
\hline $\mathrm{P} 1$ & 0 & 0 & 0 & 2 & 0 & 0 & 0 \\
\hline $\mathrm{P} 1$ & 0 & 0 & 0 & 0 & 0 & 3 & 0 \\
\hline $\mathrm{P} 1$ & 0 & 0 & 0 & 2 & 0 & 0 & 0 \\
\hline $\mathrm{P} 1$ & 0 & 0 & 0 & 0 & 0 & 0 & 0 \\
\hline $\mathrm{P} 1$ & 0 & 0 & 0 & 0 & 1 & 3 & 0 \\
\hline T0 & 0 & 0 & 0 & 0 & 0 & 6 & 0 \\
\hline T0 & 0 & 0 & 0 & 0 & 0 & 6 & 0 \\
\hline T0 & 1 & 0 & 0 & 1 & 0 & 6 & 1 \\
\hline T0 & 0 & 0 & 0 & 1 & 0 & 3 & 0 \\
\hline $\mathrm{T} 1$ & 0 & 0 & 0 & 0 & 0 & 0 & 0 \\
\hline $\mathrm{T} 1$ & 0 & 0 & 0 & 0 & 0 & 0 & 0 \\
\hline $\mathrm{T} 1$ & 0 & 1 & 0 & 0 & 0 & 0 & 0 \\
\hline $\mathrm{T} 1$ & 0 & 0 & 0 & 1 & 0 & 1 & 0 \\
\hline $\mathrm{T} 2$ & 0 & 0 & 0 & 1 & 0 & 2 & 2 \\
\hline $\mathrm{T} 2$ & 0 & 0 & 0 & 0 & 0 & 2 & 1 \\
\hline $\mathrm{T} 3$ & 0 & 0 & 0 & 2 & 0 & 0 & 0 \\
\hline $\mathrm{T} 3$ & 0 & 0 & 0 & 2 & 0 & 0 & 0 \\
\hline T3 & 0 & 0 & 1 & 2 & 0 & 0 & 0 \\
\hline
\end{tabular}


Table S5. continued ( 3 of 11)

\begin{tabular}{|c|c|c|c|c|c|c|c|}
\hline Station & $\begin{array}{c}\text { Cylichna } \\
\text { cylindracea }\end{array}$ & $\begin{array}{l}\text { Decapoda } \\
\text { zoea }\end{array}$ & $\begin{array}{l}\text { Devonia } \\
\text { perrieri }\end{array}$ & $\begin{array}{l}\text { Diplocirrus } \\
\text { glaucus }\end{array}$ & Dorvillea & $\begin{array}{l}\text { Echinocardium } \\
\text { cordatum }\end{array}$ & $\begin{array}{l}\text { Eudorella } \\
\text { truncatula }\end{array}$ \\
\hline $\mathrm{P} 0$ & 0 & 0 & 0 & 4 & 0 & 2 & 0 \\
\hline $\mathrm{P} 0$ & 0 & 0 & 0 & 1 & 0 & 0 & 1 \\
\hline $\mathrm{P} 0$ & 1 & 0 & 0 & 3 & 0 & 0 & 0 \\
\hline $\mathrm{P} 1$ & 0 & 0 & 0 & 0 & 0 & 1 & 0 \\
\hline $\mathrm{P} 1$ & 0 & 0 & 0 & 0 & 0 & 0 & 1 \\
\hline $\mathrm{P} 1$ & 0 & 0 & 0 & 0 & 1 & 0 & 0 \\
\hline $\mathrm{P} 1$ & 0 & 0 & 0 & 1 & 0 & 0 & 0 \\
\hline $\mathrm{P} 1$ & 0 & 0 & 1 & 3 & 0 & 0 & 0 \\
\hline $\mathrm{P} 1$ & 0 & 0 & 0 & 4 & 0 & 0 & 0 \\
\hline $\mathrm{P} 1$ & 0 & 0 & 0 & 1 & 0 & 1 & 0 \\
\hline T0 & 0 & 1 & 0 & 3 & 0 & 0 & 2 \\
\hline T0 & 0 & 1 & 0 & 2 & 0 & 0 & 0 \\
\hline T0 & 0 & 0 & 0 & 0 & 0 & 0 & 0 \\
\hline T0 & 0 & 0 & 0 & 1 & 0 & 1 & 0 \\
\hline $\mathrm{T} 1$ & 0 & 0 & 0 & 2 & 0 & 2 & 0 \\
\hline $\mathrm{T} 1$ & 0 & 0 & 0 & 1 & 0 & 0 & 1 \\
\hline $\mathrm{T} 1$ & 0 & 0 & 0 & 3 & 0 & 1 & 0 \\
\hline $\mathrm{T} 1$ & 0 & 0 & 0 & 0 & 0 & 0 & 0 \\
\hline $\mathrm{T} 2$ & 0 & 0 & 0 & 0 & 0 & 0 & 0 \\
\hline $\mathrm{T} 2$ & 0 & 0 & 0 & 0 & 0 & 0 & 0 \\
\hline T3 & 0 & 0 & 0 & 0 & 0 & 0 & 0 \\
\hline $\mathrm{T} 3$ & 0 & 0 & 0 & 1 & 0 & 0 & 0 \\
\hline $\mathrm{T} 3$ & 0 & 0 & 0 & 0 & 0 & 0 & 0 \\
\hline
\end{tabular}

Table S5. continued ( 4 of 11 )

\begin{tabular}{cccccccc}
\hline Station & Glycera juv. & $\begin{array}{c}\text { Glycera } \\
\text { alba }\end{array}$ & $\begin{array}{c}\text { Glycera } \\
\text { unicornis }\end{array}$ & $\begin{array}{c}\text { Glycinde } \\
\text { nordmanni }\end{array}$ & $\begin{array}{c}\text { Goniada } \\
\text { juv. }\end{array}$ & $\begin{array}{c}\text { Goniada } \\
\text { maculata }\end{array}$ & $\begin{array}{c}\text { Gyge } \\
\text { branchialis }\end{array}$ \\
\hline P0 & 0 & 0 & 0 & 0 & 0 & 0 & 0 \\
P0 & 0 & 0 & 0 & 0 & 0 & 0 & 0 \\
P0 & 0 & 0 & 0 & 0 & 0 & 0 & 0 \\
P1 & 0 & 0 & 0 & 0 & 0 & 0 & 0 \\
P1 & 1 & 0 & 0 & 0 & 0 & 0 & 0 \\
P1 & 0 & 0 & 0 & 1 & 0 & 0 & 0 \\
P1 & 0 & 0 & 0 & 0 & 0 & 0 & 0 \\
P1 & 0 & 0 & 0 & 0 & 0 & 0 & 0 \\
P1 & 1 & 0 & 0 & 0 & 0 & 0 & 0 \\
P1 & 0 & 0 & 0 & 1 & 0 & 0 & 0 \\
T0 & 0 & 0 & 0 & 0 & 0 & 0 & 0 \\
T0 & 0 & 1 & 1 & 0 & 0 & 0 & 0 \\
T0 & 0 & 1 & 0 & 0 & 0 & 0 & 0 \\
T0 & 0 & 0 & 0 & 0 & 0 & 0 & 0 \\
T1 & 0 & 0 & 0 & 0 & 0 & 0 \\
T1 & 0 & 0 & 0 & 0 & 0 & 0 \\
T1 & 0 & 0 & 0 & 0 & 0 & 0 \\
T1 & 0 & 0 & 0 & 0 & 0 & 0 \\
T2 & 0 & 0 & 0 & 0 & 0 & 0 & 0 \\
T2 & 0 & 0 & 0 & 0 & 0 & 0 & 0 \\
T3 & 0 & 0 & 0 & 0 & 0 & 0 \\
T3 & 0 & 0 & 0 & 0 & 0 & 0 \\
T3 & 0 & 0 & 0 & 0 & 0 \\
\hline
\end{tabular}


Table S5. continued (5 of 11)

\begin{tabular}{|c|c|c|c|c|c|c|c|}
\hline Station & $\begin{array}{l}\text { Hyala } \\
\text { vitrea }\end{array}$ & $\begin{array}{c}\text { Ione } \\
\text { thoracica }\end{array}$ & $\begin{array}{l}\text { Kurtiella } \\
\text { bidentata }\end{array}$ & $\begin{array}{c}\text { Leptosynapta } \\
\text { inhaerens }\end{array}$ & $\begin{array}{l}\text { Lumbrineridae } \\
\text { juv. }\end{array}$ & $\begin{array}{c}\text { Lumbrineris } \\
\text { cingulata }\end{array}$ & $\begin{array}{l}\text { Malmgrenia } \\
\text { andreapolis }\end{array}$ \\
\hline $\mathrm{P} 0$ & 0 & 0 & 7 & 0 & 0 & 4 & 0 \\
\hline $\mathrm{P} 0$ & 0 & 0 & 5 & 0 & 0 & 1 & 0 \\
\hline $\mathrm{P} 0$ & 0 & 0 & 4 & 0 & 0 & 3 & 0 \\
\hline $\mathrm{P} 1$ & 0 & 0 & 17 & 0 & 0 & 3 & 0 \\
\hline $\mathrm{P} 1$ & 0 & 0 & 6 & 0 & 1 & 4 & 0 \\
\hline $\mathrm{P} 1$ & 0 & 0 & 3 & 0 & 0 & 0 & 0 \\
\hline $\mathrm{P} 1$ & 0 & 0 & 11 & 0 & 0 & 6 & 0 \\
\hline $\mathrm{P} 1$ & 0 & 0 & 2 & 1 & 0 & 6 & 1 \\
\hline $\mathrm{P} 1$ & 0 & 0 & 1 & 0 & 2 & 5 & 0 \\
\hline $\mathrm{P} 1$ & 0 & 0 & 1 & 0 & 1 & 3 & 0 \\
\hline T0 & 0 & 0 & 6 & 0 & 0 & 4 & 0 \\
\hline T0 & 0 & 0 & 13 & 0 & 1 & 7 & 0 \\
\hline T0 & 0 & 0 & 22 & 0 & 1 & 4 & 0 \\
\hline T0 & 0 & 0 & 1 & 0 & 0 & 7 & 0 \\
\hline $\mathrm{T} 1$ & 0 & 0 & 2 & 0 & 0 & 2 & 0 \\
\hline $\mathrm{T} 1$ & 0 & 0 & 15 & 0 & 2 & 2 & 0 \\
\hline $\mathrm{T} 1$ & 0 & 0 & 6 & 0 & 3 & 2 & 0 \\
\hline $\mathrm{T} 1$ & 0 & 0 & 1 & 0 & 0 & 0 & 0 \\
\hline $\mathrm{T} 2$ & 1 & 0 & 1 & 0 & 0 & 0 & 0 \\
\hline $\mathrm{T} 2$ & 0 & 0 & 1 & 0 & 0 & 1 & 0 \\
\hline T3 & 0 & 0 & 2 & 0 & 0 & 1 & 0 \\
\hline T3 & 0 & 1 & 0 & 0 & 0 & 0 & 0 \\
\hline $\mathrm{T} 3$ & 0 & 0 & 3 & 0 & 0 & 0 & 0 \\
\hline
\end{tabular}

Table S5. continued (6 of 11)

\begin{tabular}{|c|c|c|c|c|c|c|c|}
\hline Station & $\begin{array}{c}\text { Malmgrenia } \\
\text { ljungmani }\end{array}$ & $\begin{array}{l}\text { Mediomastus } \\
\text { fragilis }\end{array}$ & Nemertea & $\begin{array}{c}\text { Nephtyidae } \\
\text { juv. }\end{array}$ & $\begin{array}{c}\text { Nephtys } \\
\text { hombergii }\end{array}$ & $\begin{array}{l}\text { Nephtys } \\
\text { incisa }\end{array}$ & $\begin{array}{l}\text { Nereididae } \\
\text { juv. }\end{array}$ \\
\hline $\mathrm{P} 0$ & 0 & 1 & 0 & 0 & 0 & 0 & 0 \\
\hline $\mathrm{P} 0$ & 0 & 0 & 2 & 0 & 1 & 0 & 1 \\
\hline P0 & 0 & 2 & 0 & 0 & 1 & 0 & 0 \\
\hline $\mathrm{P} 1$ & 0 & 0 & 1 & 0 & 1 & 0 & 0 \\
\hline $\mathrm{P} 1$ & 0 & 0 & 2 & 1 & 0 & 0 & 0 \\
\hline $\mathrm{P} 1$ & 0 & 2 & 0 & 0 & 0 & 0 & 0 \\
\hline $\mathrm{P} 1$ & 0 & 1 & 1 & 0 & 0 & 0 & 0 \\
\hline $\mathrm{P} 1$ & 0 & 0 & 0 & 0 & 0 & 0 & 0 \\
\hline $\mathrm{P} 1$ & 0 & 0 & 0 & 0 & 0 & 0 & 0 \\
\hline $\mathrm{P} 1$ & 0 & 0 & 0 & 0 & 0 & 0 & 0 \\
\hline T0 & 1 & 0 & 0 & 0 & 1 & 0 & 0 \\
\hline T0 & 0 & 0 & 5 & 0 & 0 & 0 & 0 \\
\hline T0 & 0 & 1 & 1 & 0 & 0 & 0 & 0 \\
\hline T0 & 0 & 1 & 1 & 1 & 0 & 0 & 0 \\
\hline $\mathrm{T} 1$ & 0 & 0 & 1 & 1 & 0 & 0 & 0 \\
\hline $\mathrm{T} 1$ & 0 & 0 & 0 & 0 & 1 & 0 & 0 \\
\hline $\mathrm{T} 1$ & 0 & 0 & 0 & 0 & 0 & 1 & 0 \\
\hline $\mathrm{T} 1$ & 0 & 0 & 1 & 0 & 1 & 0 & 0 \\
\hline $\mathrm{T} 2$ & 0 & 0 & 0 & 0 & 0 & 0 & 0 \\
\hline $\mathrm{T} 2$ & 0 & 0 & 0 & 0 & 0 & 0 & 0 \\
\hline T3 & 0 & 0 & 0 & 0 & 0 & 0 & 0 \\
\hline $\mathrm{T} 3$ & 0 & 0 & 0 & 0 & 0 & 0 & 0 \\
\hline $\mathrm{T} 3$ & 0 & 0 & 0 & 0 & 0 & 0 & 0 \\
\hline
\end{tabular}


Table S5. continued ( 7 of 11)

\begin{tabular}{|c|c|c|c|c|c|c|c|}
\hline Station & Notomastus & Nucula juv. & $\begin{array}{l}\text { Nucula } \\
\text { nitidosa }\end{array}$ & $\begin{array}{l}\text { Ophiura } \\
\text { albida }\end{array}$ & $\begin{array}{l}\text { Ophiuroidea } \\
\text { juv. }\end{array}$ & $\begin{array}{l}\text { Oxydromus } \\
\text { flexиоsиs }\end{array}$ & $\begin{array}{c}\text { Parexogone } \\
\text { hebes }\end{array}$ \\
\hline P0 & 0 & 2 & 0 & 0 & 27 & 3 & 0 \\
\hline $\mathrm{P} 0$ & 0 & 4 & 0 & 1 & 8 & 1 & 0 \\
\hline P0 & 0 & 3 & 0 & 1 & 17 & 0 & 0 \\
\hline $\mathrm{P} 1$ & 0 & 3 & 1 & 0 & 1 & 2 & 1 \\
\hline $\mathrm{P} 1$ & 0 & 2 & 1 & 0 & 2 & 1 & 0 \\
\hline $\mathrm{P} 1$ & 0 & 3 & 1 & 0 & 6 & 0 & 0 \\
\hline $\mathrm{P} 1$ & 0 & 4 & 0 & 0 & 2 & 0 & 0 \\
\hline $\mathrm{P} 1$ & 2 & 2 & 1 & 1 & 3 & 1 & 0 \\
\hline $\mathrm{P} 1$ & 1 & 3 & 1 & 1 & 6 & 1 & 0 \\
\hline $\mathrm{P} 1$ & 2 & 2 & 1 & 0 & 6 & 0 & 0 \\
\hline T0 & 0 & 2 & 0 & 0 & 27 & 0 & 1 \\
\hline T0 & 0 & 5 & 0 & 0 & 24 & 0 & 0 \\
\hline T0 & 0 & 3 & 3 & 0 & 19 & 0 & 0 \\
\hline T0 & 1 & 3 & 1 & 0 & 20 & 0 & 0 \\
\hline $\mathrm{T} 1$ & 0 & 2 & 0 & 0 & 7 & 0 & 0 \\
\hline $\mathrm{T} 1$ & 1 & 2 & 0 & 0 & 6 & 0 & 0 \\
\hline $\mathrm{T} 1$ & 0 & 2 & 1 & 0 & 8 & 0 & 0 \\
\hline $\mathrm{T} 1$ & 0 & 2 & 0 & 0 & 1 & 0 & 0 \\
\hline $\mathrm{T} 2$ & 0 & 2 & 0 & 1 & 10 & 0 & 0 \\
\hline $\mathrm{T} 2$ & 1 & 2 & 0 & 1 & 10 & 1 & 0 \\
\hline T3 & 0 & 2 & 0 & 0 & 20 & 0 & 0 \\
\hline T3 & 0 & 2 & 0 & 0 & 0 & 0 & 0 \\
\hline $\mathrm{T} 3$ & 0 & 2 & 0 & 0 & 0 & 0 & 0 \\
\hline
\end{tabular}

Table S5. continued (8 of 11)

\begin{tabular}{|c|c|c|c|c|c|c|c|}
\hline Station & $\begin{array}{c}\text { Pariambus } \\
\text { typicus }\end{array}$ & $\begin{array}{c}\text { Perioculodes } \\
\text { longimanus }\end{array}$ & $\begin{array}{c}\text { Phaxas } \\
\text { pellucidus } \\
\text { juv. }\end{array}$ & $\begin{array}{l}\text { Philinidae } \\
\text { juv. }\end{array}$ & $\begin{array}{l}\text { Pholoe } \\
\text { baltica }\end{array}$ & Phoronida & $\begin{array}{c}\text { Podarkeopsis } \\
\text { capensis }\end{array}$ \\
\hline $\mathrm{P} 0$ & 0 & 0 & 0 & 0 & 3 & 0 & 2 \\
\hline $\mathrm{P} 0$ & 1 & 0 & 1 & 0 & 0 & 0 & 2 \\
\hline $\mathrm{P} 0$ & 0 & 0 & 2 & 0 & 0 & 1 & 2 \\
\hline $\mathrm{P} 1$ & 0 & 0 & 1 & 0 & 3 & 0 & 1 \\
\hline $\mathrm{P} 1$ & 0 & 0 & 0 & 0 & 0 & 0 & 0 \\
\hline $\mathrm{P} 1$ & 0 & 0 & 0 & 0 & 2 & 0 & 2 \\
\hline $\mathrm{P} 1$ & 0 & 0 & 0 & 0 & 0 & 0 & 1 \\
\hline $\mathrm{P} 1$ & 0 & 0 & 0 & 0 & 0 & 0 & 0 \\
\hline $\mathrm{P} 1$ & 0 & 0 & 0 & 0 & 1 & 0 & 2 \\
\hline $\mathrm{P} 1$ & 0 & 0 & 1 & 0 & 1 & 0 & 0 \\
\hline T0 & 0 & 1 & 0 & 0 & 0 & 0 & 0 \\
\hline T0 & 0 & 0 & 0 & 0 & 1 & 0 & 1 \\
\hline T0 & 0 & 0 & 0 & 0 & 0 & 2 & 1 \\
\hline T0 & 0 & 0 & 0 & 0 & 0 & 1 & 0 \\
\hline $\mathrm{T} 1$ & 0 & 0 & 1 & 0 & 1 & 3 & 0 \\
\hline $\mathrm{T} 1$ & 0 & 0 & 0 & 1 & 1 & 6 & 0 \\
\hline $\mathrm{T} 1$ & 0 & 0 & 0 & 1 & 0 & 0 & 1 \\
\hline $\mathrm{T} 1$ & 0 & 0 & 0 & 0 & 0 & 1 & 1 \\
\hline $\mathrm{T} 2$ & 1 & 0 & 0 & 0 & 0 & 0 & 0 \\
\hline $\mathrm{T} 2$ & 0 & 0 & 0 & 0 & 1 & 0 & 0 \\
\hline T3 & 0 & 0 & 0 & 0 & 0 & 1 & 0 \\
\hline T3 & 0 & 0 & 0 & 0 & 0 & 1 & 0 \\
\hline $\mathrm{T} 3$ & 0 & 0 & 0 & 0 & 0 & 1 & 0 \\
\hline
\end{tabular}


Table S5. continued ( 9 of 11)

\begin{tabular}{|c|c|c|c|c|c|c|}
\hline Station & Podocopida & $\begin{array}{c}\text { Prionospio } \\
\text { multibranchiata }\end{array}$ & Pseudocumatidae & $\begin{array}{c}\text { Scalibregma } \\
\text { inflatum }\end{array}$ & $\begin{array}{l}\text { Sigalionidae } \\
\text { juv. }\end{array}$ & $\begin{array}{c}\text { Spatangoida } \\
\text { juv. }\end{array}$ \\
\hline $\mathrm{P} 0$ & 1 & 0 & 0 & 1 & 0 & 1 \\
\hline $\mathrm{P} 0$ & 0 & 0 & 0 & 4 & 0 & 3 \\
\hline $\mathrm{P} 0$ & 0 & 2 & 1 & 3 & 0 & 8 \\
\hline $\mathrm{P} 1$ & 0 & 0 & 0 & 0 & 0 & 0 \\
\hline $\mathrm{P} 1$ & 0 & 1 & 0 & 0 & 0 & 1 \\
\hline $\mathrm{P} 1$ & 0 & 3 & 0 & 3 & 0 & 1 \\
\hline $\mathrm{P} 1$ & 0 & 0 & 0 & 2 & 0 & 0 \\
\hline $\mathrm{P} 1$ & 0 & 1 & 0 & 2 & 0 & 0 \\
\hline $\mathrm{P} 1$ & 0 & 0 & 0 & 1 & 0 & 1 \\
\hline $\mathrm{P} 1$ & 0 & 0 & 0 & 1 & 1 & 3 \\
\hline T0 & 0 & 1 & 0 & 1 & 0 & 18 \\
\hline T0 & 0 & 1 & 0 & 2 & 0 & 3 \\
\hline T0 & 0 & 0 & 0 & 1 & 0 & 2 \\
\hline T0 & 0 & 3 & 0 & 3 & 0 & 7 \\
\hline $\mathrm{T} 1$ & 0 & 0 & 0 & 2 & 0 & 3 \\
\hline $\mathrm{T} 1$ & 0 & 0 & 0 & 3 & 0 & 9 \\
\hline $\mathrm{T} 1$ & 0 & 0 & 0 & 3 & 0 & 12 \\
\hline $\mathrm{T} 1$ & 0 & 0 & 0 & 0 & 0 & 1 \\
\hline $\mathrm{T} 2$ & 0 & 0 & 0 & 0 & 0 & 1 \\
\hline $\mathrm{T} 2$ & 0 & 0 & 0 & 1 & 0 & 2 \\
\hline T3 & 0 & 0 & 0 & 0 & 0 & 1 \\
\hline T3 & 0 & 0 & 0 & 0 & 0 & 0 \\
\hline $\mathrm{T} 3$ & 0 & 0 & 0 & 0 & 0 & 0 \\
\hline
\end{tabular}

Table S5. continued (10 of 11)

\begin{tabular}{ccccccc}
\hline Station & $\begin{array}{c}\text { Spiophanes } \\
\text { bombyx }\end{array}$ & $\begin{array}{c}\text { Spisula juv. } \\
\text { Sthenelais } \\
\text { juv. }\end{array}$ & $\begin{array}{c}\text { Sthenelais } \\
\text { limicola }\end{array}$ & $\begin{array}{c}\text { Tellimya } \\
\text { ferruginosa }\end{array}$ & Tellinoidea juv. \\
\hline P0 & 0 & 0 & 0 & 0 & 14 & 0 \\
P0 & 1 & 0 & 0 & 0 & 2 & 0 \\
P0 & 0 & 0 & 2 & 0 & 0 & 1 \\
P1 & 0 & 0 & 0 & 0 & 0 & 0 \\
P1 & 0 & 0 & 0 & 0 & 0 & 0 \\
P1 & 0 & 0 & 0 & 0 & 0 & 0 \\
P1 & 0 & 0 & 0 & 0 & 1 & 0 \\
P1 & 0 & 0 & 0 & 0 & 0 & 0 \\
P1 & 0 & 0 & 0 & 0 & 0 & 0 \\
P1 & 0 & 0 & 0 & 0 & 0 & 0 \\
T0 & 1 & 0 & 1 & 0 & 0 & 0 \\
T0 & 2 & 0 & 0 & 0 & 1 & 0 \\
T0 & 0 & 1 & 0 & 0 & 0 & 0 \\
T0 & 0 & 0 & 0 & 0 & 0 & 0 \\
T1 & 0 & 0 & 0 & 0 & 0 & 0 \\
T1 & 0 & 1 & 0 & 0 & 1 & 0 \\
T1 & 0 & 0 & 0 & 0 & 2 & 0 \\
T1 & 0 & 0 & 0 & 0 & 0 & 0 \\
T2 & 0 & 0 & 0 & 0 & 1 & 0 \\
T2 & 0 & 0 & 0 & 0 & 0 & 0 \\
T3 & 0 & 0 & 0 & 0 & 0 & 0 \\
T3 & 0 & 0 & 0 & 0 & 0 & 0 \\
T3 & 0 & 0 & 0 & 0 & 0 & 0 \\
\hline
\end{tabular}


Table S5. continued (11 of 11)

\begin{tabular}{ccccccc}
\hline Station & $\begin{array}{c}\text { Thracia } \\
\text { convexa }\end{array}$ & $\begin{array}{c}\text { Thracioidea } \\
\text { juv. }\end{array}$ & $\begin{array}{c}\text { Thyasira } \\
\text { flexuosa }\end{array}$ & $\begin{array}{c}\text { Thysanocardia } \\
\text { procera }\end{array}$ & $\begin{array}{c}\text { Turbellaria } \\
\text { Upogebia } \\
\text { deltaura }\end{array}$ \\
\hline P0 & 1 & 1 & 0 & 0 & 0 & 0 \\
P0 & 0 & 1 & 0 & 0 & 0 & 0 \\
P0 & 0 & 0 & 1 & 0 & 0 & 0 \\
P1 & 0 & 0 & 0 & 1 & 0 & 0 \\
P1 & 0 & 0 & 0 & 0 & 0 & 0 \\
P1 & 0 & 0 & 1 & 2 & 0 & 0 \\
P1 & 0 & 0 & 0 & 0 & 0 & 0 \\
P1 & 0 & 1 & 0 & 0 & 0 & 0 \\
P1 & 0 & 0 & 0 & 0 & 0 & 0 \\
P1 & 0 & 0 & 0 & 1 & 0 & 0 \\
T0 & 0 & 2 & 0 & 0 & 0 & 0 \\
T0 & 0 & 0 & 0 & 0 & 0 & 0 \\
T0 & 0 & 0 & 0 & 0 & 0 & 0 \\
T0 & 0 & 0 & 1 & 0 & 0 & 0 \\
T1 & 0 & 0 & 0 & 0 & 0 & 0 \\
T1 & 0 & 2 & 0 & 0 & 0 & 0 \\
T1 & 0 & 0 & 0 & 0 & 0 \\
T1 & 0 & 0 & 0 & 0 & 0 & 0 \\
T2 & 0 & 0 & 0 & 0 & 0 & 0 \\
T2 & 0 & 0 & 0 & 0 & 0 & 0 \\
T3 & 0 & 0 & 0 & 0 & 0 \\
T3 & 1 & 0 & 0 & 0 & 0 & 0 \\
T3 & 0 & 0 & 0 & 0 & 0 \\
\hline
\end{tabular}


Table S6. (1 of 13)

Macrofauna individual densities found in deep cores.

\begin{tabular}{|c|c|c|c|c|c|c|c|c|}
\hline Station & $\begin{array}{l}\text { Abra } \\
\text { alba }\end{array}$ & $\begin{array}{l}\text { Abra } \\
\text { nitida }\end{array}$ & $\begin{array}{l}\text { Abra } \\
\text { juv. }\end{array}$ & $\begin{array}{l}\text { Amphiura } \\
\text { filiformis }\end{array}$ & Aphroditidae & $\begin{array}{c}\text { Argissa } \\
\text { hamatipes }\end{array}$ & Atherospio & $\begin{array}{c}\text { Bathyporeia } \\
\text { guilliamsoniana }\end{array}$ \\
\hline $\mathrm{T} 1$ & 0 & 0 & 62 & 34 & 6 & 0 & 0 & 0 \\
\hline $\mathrm{T} 1$ & 0 & 0 & 335 & 54 & 1 & 0 & 0 & 0 \\
\hline $\mathrm{P} 0$ & 0 & 0 & 177 & 15 & 0 & 0 & 4 & 0 \\
\hline P0 & 0 & 0 & 62 & 30 & 0 & 0 & 0 & 0 \\
\hline P0 & 2 & 1 & 202 & 32 & 1 & 0 & 2 & 0 \\
\hline P0 & 0 & 0 & 110 & 19 & 1 & 0 & 8 & 0 \\
\hline $\mathrm{P} 0$ & 0 & 0 & 403 & 37 & 2 & 0 & 7 & 0 \\
\hline Ref & 0 & 0 & 140 & 17 & 0 & 1 & 11 & 0 \\
\hline Ref & 2 & 0 & 403 & 36 & 3 & 0 & 6 & 0 \\
\hline Ref & 0 & 0 & 208 & 15 & 3 & 0 & 1 & 0 \\
\hline $\mathrm{P} 1$ & 7 & 0 & 26 & 32 & 0 & 0 & 10 & 0 \\
\hline $\mathrm{P} 1$ & 0 & 0 & 35 & 50 & 2 & 0 & 31 & 0 \\
\hline $\mathrm{P} 1$ & 0 & 0 & 20 & 12 & 1 & 0 & 0 & 0 \\
\hline $\mathrm{P} 1$ & 0 & 0 & 31 & 40 & 0 & 0 & 14 & 1 \\
\hline
\end{tabular}

Table S6. continued ( 2 of 13 )

\begin{tabular}{|c|c|c|c|c|c|c|c|}
\hline Station & Bopyridae & Brachyura & $\begin{array}{l}\text { Callianassa } \\
\text { subterranea }\end{array}$ & Caprellidae & $\begin{array}{c}\text { Chamelea } \\
\text { striatula }\end{array}$ & $\begin{array}{c}\text { Corbula } \\
\text { gibba }\end{array}$ & $\begin{array}{c}\text { Corystes } \\
\text { cassivelaunus }\end{array}$ \\
\hline $\mathrm{T} 1$ & 0 & 0 & 9 & 0 & 0 & 6 & 2 \\
\hline $\mathrm{T} 1$ & 0 & 0 & 4 & 0 & 0 & 3 & 1 \\
\hline $\mathrm{P} 0$ & 0 & 0 & 7 & 0 & 0 & 9 & 2 \\
\hline $\mathrm{P} 0$ & 0 & 0 & 10 & 0 & 0 & 0 & 0 \\
\hline $\mathrm{P} 0$ & 0 & 0 & 7 & 0 & 0 & 9 & 1 \\
\hline $\mathrm{P} 0$ & 0 & 2 & 10 & 0 & 0 & 17 & 1 \\
\hline $\mathrm{P} 0$ & 0 & 0 & 4 & 0 & 0 & 0 & 0 \\
\hline Ref & 0 & 0 & 8 & 0 & 0 & 12 & 4 \\
\hline Ref & 0 & 1 & 5 & 0 & 0 & 21 & 0 \\
\hline Ref & 4 & 0 & 9 & 0 & 0 & 3 & 1 \\
\hline $\mathrm{P} 1$ & 0 & 0 & 10 & 0 & 0 & 4 & 0 \\
\hline $\mathrm{P} 1$ & 0 & 0 & 6 & 0 & 0 & 4 & 0 \\
\hline $\mathrm{P} 1$ & 0 & 0 & 8 & 0 & 1 & 11 & 0 \\
\hline $\mathrm{P} 1$ & 2 & 0 & 9 & 0 & 0 & 5 & 1 \\
\hline
\end{tabular}

Table S6. continued ( 3 of 13)

\begin{tabular}{|c|c|c|c|c|c|c|c|}
\hline Station & $\begin{array}{l}\text { Crangon } \\
\text { crangon }\end{array}$ & $\begin{array}{c}\text { Cylichna } \\
\text { cylindracea }\end{array}$ & $\begin{array}{l}\text { Decapoda } \\
\text { juv. }\end{array}$ & $\begin{array}{l}\text { Devonia } \\
\text { perrieri }\end{array}$ & $\begin{array}{l}\text { Diplocirrus } \\
\text { glaucus }\end{array}$ & $\begin{array}{l}\text { Echinocardium } \\
\text { cordatum }\end{array}$ & Echinoida juv. \\
\hline $\mathrm{T} 1$ & 0 & 0 & 0 & 0 & 8 & 3 & 6 \\
\hline $\mathrm{T} 1$ & 0 & 0 & 0 & 0 & 5 & 0 & 6 \\
\hline P0 & 0 & 0 & 1 & 0 & 21 & 1 & 9 \\
\hline P0 & 0 & 0 & 0 & 0 & 5 & 0 & 2 \\
\hline $\mathrm{P} 0$ & 0 & 0 & 0 & 0 & 10 & 2 & 13 \\
\hline $\mathrm{P} 0$ & 0 & 1 & 0 & 0 & 8 & 4 & 0 \\
\hline $\mathrm{P} 0$ & 0 & 1 & 0 & 0 & 9 & 2 & 0 \\
\hline Ref & 1 & 1 & 0 & 0 & 28 & 3 & 0 \\
\hline Ref & 0 & 0 & 0 & 0 & 16 & 3 & 7 \\
\hline Ref & 0 & 0 & 0 & 1 & 9 & 2 & 24 \\
\hline $\mathrm{P} 1$ & 0 & 1 & 0 & 0 & 0 & 0 & 1 \\
\hline $\mathrm{P} 1$ & 0 & 0 & 0 & 0 & 2 & 1 & 1 \\
\hline $\mathrm{P} 1$ & 0 & 0 & 0 & 0 & 1 & 0 & 1 \\
\hline $\mathrm{P} 1$ & 0 & 0 & 0 & 0 & 1 & 2 & 0 \\
\hline
\end{tabular}


Table S6. continued (4 of 13)

\begin{tabular}{cccccccc}
\hline Station & $\begin{array}{c}\text { Eudorella } \\
\text { truncatula }\end{array}$ & $\begin{array}{c}\text { Eunereis } \\
\text { longissima }\end{array}$ & $\begin{array}{c}\text { Fabulina } \\
\text { fabula juv. }\end{array}$ & $\begin{array}{c}\text { Gammaroidea } \\
\text { juv. }\end{array}$ & $\begin{array}{c}\text { Gastrosaccus } \\
\text { sanctus }\end{array}$ & $\begin{array}{c}\text { Gattyana } \\
\text { cirrhosa }\end{array}$ & Glycera alba \\
\hline T1 & 3 & 0 & 0 & 0 & 0 & 0 & 1 \\
T1 & 3 & 1 & 0 & 0 & 0 & 0 & 0 \\
P0 & 0 & 0 & 0 & 2 & 0 & 0 & 0 \\
P0 & 0 & 0 & 0 & 0 & 0 & 0 & 0 \\
P0 & 6 & 0 & 0 & 0 & 0 & 0 & 0 \\
P0 & 0 & 2 & 0 & 0 & 0 & 0 & 0 \\
P0 & 0 & 2 & 1 & 0 & 0 & 0 & 0 \\
Ref & 1 & 1 & 0 & 0 & 0 & 0 & 0 \\
Ref & 10 & 2 & 0 & 0 & 0 & 0 & 0 \\
Ref & 2 & 1 & 0 & 0 & 0 & 0 & 0 \\
P1 & 0 & 1 & 0 & 0 & 0 & 0 & 0 \\
P1 & 0 & 2 & 0 & 0 & 0 & 0 & 0 \\
P1 & 0 & 2 & 0 & 0 & 0 & 0 & 0 \\
P1 & 0 & 2 & 0 & 0 & 0 & 0 \\
\hline
\end{tabular}

Table S6. continued (5 of 13)

\begin{tabular}{cccccccc}
\hline Station & $\begin{array}{c}\text { Glycera } \\
\text { juv. }\end{array}$ & $\begin{array}{c}\text { Glycinde } \\
\text { nordmanni }\end{array}$ & $\begin{array}{c}\text { Goneplax } \\
\text { rhomboides }\end{array}$ & $\begin{array}{c}\text { Goniada } \\
\text { maculata }\end{array}$ & $\begin{array}{c}\text { Goniadidae } \\
\text { juv. }\end{array}$ & Holothuroidea & Hyala vitrea \\
\hline T1 & 0 & 0 & 0 & 0 & 0 & 0 & 0 \\
T1 & 0 & 0 & 0 & 0 & 0 & 0 & 0 \\
P0 & 3 & 0 & 0 & 0 & 0 & 0 & 0 \\
P0 & 0 & 0 & 0 & 0 & 3 & 0 & 0 \\
P0 & 0 & 1 & 0 & 1 & 0 & 1 & 0 \\
P0 & 1 & 0 & 0 & 0 & 0 & 0 & 0 \\
P0 & 0 & 0 & 1 & 0 & 4 & 1 & 0 \\
Ref & 0 & 1 & 0 & 1 & 1 & 0 & 0 \\
Ref & 0 & 1 & 0 & 0 & 1 & 0 & 0 \\
Ref & 0 & 0 & 0 & 1 & 0 & 1 & 0 \\
P1 & 0 & 0 & 0 & 0 & 0 & 0 & 0 \\
P1 & 0 & 0 & 0 & 0 & 0 & 0 & 0 \\
P1 & 0 & 0 & 0 & 0 & 3 & 0 & 1 \\
P1 & 0 & 0 & 0 & & & 0 \\
\hline
\end{tabular}

Table S6. continued (6 of 13)

\begin{tabular}{cccccccc}
\hline Station & Isopoda & $\begin{array}{c}\text { Kurtiella } \\
\text { bidentata }\end{array}$ & $\begin{array}{c}\text { Lepton } \\
\text { squamosum }\end{array}$ & $\begin{array}{c}\text { Leucothoe } \\
\text { incisa }\end{array}$ & $\begin{array}{c}\text { Leucothoe } \\
\text { lilljeborgi }\end{array}$ & $\begin{array}{c}\text { Leucothoe } \\
\text { procera }\end{array}$ & $\begin{array}{c}\text { Lumbrineris } \\
\text { T1 }\end{array}$ \\
\hline 0 & 13 & 0 & 0 & 0 & 0 & 19 \\
T1 & 0 & 43 & 0 & 0 & 0 & 0 & 13 \\
P0 & 0 & 11 & 0 & 0 & 0 & 0 & 29 \\
P0 & 0 & 9 & 0 & 0 & 0 & 0 & 19 \\
P0 & 0 & 6 & 0 & 0 & 1 & 0 & 22 \\
P0 & 1 & 10 & 0 & 1 & 0 & 0 & 11 \\
P0 & 0 & 7 & 0 & 0 & 0 & 0 & 37 \\
Ref & 0 & 9 & 0 & 0 & 0 & 0 & 30 \\
Ref & 0 & 21 & 0 & 0 & 0 & 0 & 25 \\
Ref & 0 & 10 & 2 & 0 & 0 & 0 & 12 \\
P1 & 0 & 7 & 0 & 1 & 0 & 0 & 18 \\
P1 & 0 & 15 & 0 & 2 & 0 & 0 & 19 \\
P1 & 0 & 5 & 0 & 0 & 0 & 0 & 7 \\
P1 & 0 & 4 & 0 & 0 & 0 & 0 \\
\hline
\end{tabular}


Table S6. continued ( 7 of 13)

\begin{tabular}{|c|c|c|c|c|c|c|c|}
\hline Station & $\begin{array}{l}\text { Malmgrenia } \\
\text { andreapolis }\end{array}$ & $\begin{array}{l}\text { Mediomastus } \\
\text { fragilis }\end{array}$ & $\begin{array}{c}\text { Megaluropus } \\
\text { agilis }\end{array}$ & $\begin{array}{l}\text { Mytilidae } \\
\text { juv. }\end{array}$ & Nemertea & $\begin{array}{l}\text { Nephtyidae } \\
\text { juv. }\end{array}$ & $\begin{array}{c}\text { Nephtys } \\
\text { hombergii }\end{array}$ \\
\hline $\mathrm{T} 1$ & 0 & 0 & 0 & 0 & 1 & 2 & 1 \\
\hline $\mathrm{T} 1$ & 0 & 1 & 0 & 0 & 1 & 1 & 1 \\
\hline P0 & 0 & 0 & 0 & 0 & 13 & 2 & 1 \\
\hline P0 & 0 & 4 & 1 & 0 & 14 & 0 & 1 \\
\hline P0 & 0 & 3 & 0 & 0 & 3 & 3 & 1 \\
\hline P0 & 0 & 2 & 3 & 0 & 2 & 4 & 1 \\
\hline P0 & 0 & 7 & 1 & 0 & 0 & 3 & 0 \\
\hline Ref & 1 & 2 & 0 & 0 & 3 & 7 & 1 \\
\hline Ref & 0 & 0 & 0 & 0 & 5 & 4 & 1 \\
\hline Ref & 0 & 0 & 0 & 0 & 0 & 1 & 0 \\
\hline $\mathrm{P} 1$ & 0 & 11 & 0 & 0 & 5 & 0 & 2 \\
\hline $\mathrm{P} 1$ & 0 & 6 & 0 & 0 & 1 & 0 & 0 \\
\hline $\mathrm{P} 1$ & 0 & 9 & 0 & 0 & 1 & 0 & 0 \\
\hline $\mathrm{P} 1$ & 0 & 7 & 0 & 0 & 4 & 3 & 0 \\
\hline
\end{tabular}

Table S6. continued ( 8 of 13)

\begin{tabular}{cccccccc}
\hline Station & $\begin{array}{c}\text { Nephtys } \\
\text { incisa }\end{array}$ & $\begin{array}{c}\text { Nereididae } \\
\text { juv. }\end{array}$ & $\begin{array}{c}\text { Notomastus } \\
\text { latericeus }\end{array}$ & $\begin{array}{c}\text { Nucula } \\
\text { nitidosa }\end{array}$ & Nucula juv. & Oligochaeta & $\begin{array}{c}\text { Ophiura } \\
\text { albida }\end{array}$ \\
\hline T1 & 0 & 1 & 1 & 3 & 0 & 0 & 2 \\
T1 & 2 & 0 & 0 & 0 & 0 & 1 & 0 \\
P0 & 0 & 0 & 1 & 0 & 0 & 7 & 0 \\
P0 & 0 & 2 & 0 & 3 & 0 & 0 & 0 \\
P0 & 0 & 0 & 0 & 3 & 5 & 0 & 0 \\
P0 & 0 & 2 & 0 & 15 & 0 & 0 & 0 \\
P0 & 0 & 1 & 1 & 3 & 0 & 0 & 0 \\
Ref & 0 & 0 & 3 & 3 & 0 & 0 & 0 \\
Ref & 0 & 0 & 2 & 1 & 0 & 0 & 0 \\
Ref & 1 & 0 & 3 & 0 & 0 & 0 & 0 \\
P1 & 0 & 0 & 0 & 3 & 0 & 0 & 0 \\
P1 & 0 & 0 & 0 & 0 & 0 & 0 & 0 \\
P1 & 0 & 0 & 1 & 6 & 0 & 0 & 0 \\
P1 & 1 & 0 & 1 & 9 & 0 & 0 \\
\hline
\end{tabular}

Table S6. continued ( 9 of 13)

\begin{tabular}{cccccccc}
\hline Station & $\begin{array}{c}\text { Ophiuroidea } \\
\text { juv. }\end{array}$ & $\begin{array}{c}\text { Oxydromus } \\
\text { flexuosus }\end{array}$ & $\begin{array}{c}\text { Parexogone } \\
\text { hebes }\end{array}$ & $\begin{array}{c}\text { Pariambus } \\
\text { typicus }\end{array}$ & $\begin{array}{c}\text { Perioculodes } \\
\text { longimanus }\end{array}$ & $\begin{array}{c}\text { Pharidae juv. } \\
\text { Phaxas } \\
\text { pellucidus }\end{array}$ \\
\hline T1 & 64 & 4 & 0 & 0 & 0 & 0 & 2 \\
T1 & 76 & 1 & 0 & 0 & 0 & 0 & 2 \\
P0 & 14 & 11 & 0 & 0 & 0 & 0 & 0 \\
P0 & 8 & 9 & 2 & 0 & 0 & 0 & 0 \\
P0 & 72 & 0 & 0 & 0 & 0 & 0 & 0 \\
P0 & 10 & 4 & 0 & 0 & 0 & 0 & 0 \\
P0 & 31 & 3 & 1 & 0 & 0 & 0 & 0 \\
Ref & 45 & 4 & 1 & 0 & 0 & 0 & 0 \\
Ref & 34 & 1 & 0 & 1 & 2 & 0 & 0 \\
Ref & 29 & 0 & 0 & 0 & 0 & 0 & 0 \\
P1 & 5 & 3 & 3 & 0 & 0 & 0 & 0 \\
P1 & 4 & 5 & 1 & 0 & 0 & 0 & 0 \\
P1 & 6 & 4 & 2 & 0 & 0 & 0 \\
P1 & 21 & 2 & 1 & 0 & 0 & 0 \\
\hline
\end{tabular}


Table S6. continued (10 of 13)

\begin{tabular}{cccccccc}
\hline Station & Philine & $\begin{array}{c}\text { Pholoe } \\
\text { baltica }\end{array}$ & Phoronida & $\begin{array}{c}\text { Podarkeopsis } \\
\text { capensis }\end{array}$ & $\begin{array}{c}\text { Polynoidae } \\
\text { juv. }\end{array}$ & $\begin{array}{c}\text { Portumnus } \\
\text { latipes }\end{array}$ & $\begin{array}{c}\text { Portumnus } \\
\text { latipes juv. }\end{array}$ \\
\hline T1 & 0 & 3 & 15 & 3 & 0 & 0 & 0 \\
T1 & 5 & 4 & 11 & 1 & 0 & 0 & 0 \\
P0 & 0 & 0 & 5 & 0 & 0 & 0 & 0 \\
P0 & 0 & 3 & 9 & 0 & 1 & 0 & 0 \\
P0 & 0 & 4 & 7 & 2 & 2 & 0 & 0 \\
P0 & 0 & 7 & 23 & 0 & 0 & 0 & 0 \\
P0 & 0 & 4 & 16 & 0 & 4 & 0 & 0 \\
Ref & 0 & 0 & 14 & 0 & 0 & 0 & 0 \\
Ref & 0 & 9 & 22 & 1 & 0 & 0 & 0 \\
Ref & 0 & 2 & 5 & 3 & 0 & 0 & 0 \\
P1 & 0 & 4 & 25 & 0 & 0 & 0 & 0 \\
P1 & 0 & 2 & 39 & 0 & 2 & 0 & 0 \\
P1 & 0 & 3 & 19 & 0 & 0 & 0 & 0 \\
P1 & 0 & 0 & 6 & 3 & 0 & 0 \\
\hline
\end{tabular}

Table S6. continued (11 of 13)

\begin{tabular}{|c|c|c|c|c|c|c|c|}
\hline Station & Prionospio & $\begin{array}{l}\text { Pseudione } \\
\text { borealis }\end{array}$ & $\begin{array}{c}\text { Pseudocuma } \\
\text { longicornis }\end{array}$ & $\begin{array}{l}\text { Pseudocuma } \\
\text { simile }\end{array}$ & $\begin{array}{c}\text { Scalibregma } \\
\text { inflatum }\end{array}$ & $\begin{array}{c}\text { Sigalionidae } \\
\text { juv. }\end{array}$ & Sipuncula \\
\hline $\mathrm{T} 1$ & 0 & 0 & 0 & 0 & 12 & 0 & 3 \\
\hline $\mathrm{T} 1$ & 4 & 0 & 0 & 0 & 4 & 0 & 0 \\
\hline $\mathrm{P} 0$ & 0 & 0 & 0 & 0 & 22 & 0 & 1 \\
\hline P0 & 0 & 0 & 1 & 0 & 17 & 0 & 1 \\
\hline P0 & 1 & 0 & 0 & 0 & 22 & 2 & 1 \\
\hline P0 & 0 & 0 & 1 & 0 & 16 & 0 & 2 \\
\hline $\mathrm{P} 0$ & 0 & 0 & 2 & 0 & 31 & 0 & 1 \\
\hline Ref & 3 & 0 & 1 & 0 & 39 & 0 & 2 \\
\hline Ref & 0 & 0 & 1 & 0 & 11 & 2 & 0 \\
\hline Ref & 0 & 0 & 0 & 0 & 6 & 0 & 0 \\
\hline $\mathrm{P} 1$ & 2 & 0 & 0 & 0 & 4 & 0 & 1 \\
\hline $\mathrm{P} 1$ & 1 & 0 & 0 & 2 & 7 & 0 & 0 \\
\hline $\mathrm{P} 1$ & 1 & 1 & 0 & 0 & 3 & 0 & 1 \\
\hline $\mathrm{P} 1$ & 9 & 0 & 2 & 0 & 8 & 1 & 2 \\
\hline
\end{tabular}

Table S6. continued (12 of 13)

\begin{tabular}{cccccccc}
\hline Station & $\begin{array}{c}\text { Spio } \\
\text { goniocephala }\end{array}$ & $\begin{array}{c}\text { Spiophanes } \\
\text { bombyx }\end{array}$ & Spisula juv. & $\begin{array}{c}\text { Tellimya } \\
\text { ferruginosa }\end{array}$ & $\begin{array}{c}\text { Tellinoidea } \\
\text { juv. }\end{array}$ & $\begin{array}{c}\text { Terebellidae } \\
\text { juv. }\end{array}$ & $\begin{array}{c}\text { Thracia } \\
\text { convexa }\end{array}$ \\
\hline T1 & 0 & 0 & 0 & 0 & 1 & 0 & 0 \\
T1 & 0 & 0 & 0 & 2 & 1 & 0 & 0 \\
P0 & 0 & 1 & 0 & 0 & 1 & 0 & 0 \\
P0 & 0 & 2 & 0 & 0 & 1 & 0 & 0 \\
P0 & 0 & 1 & 0 & 2 & 0 & 0 & 0 \\
P0 & 0 & 1 & 0 & 4 & 0 & 0 & 0 \\
P0 & 0 & 0 & 1 & 1 & 2 & 0 & 0 \\
Ref & 0 & 1 & 0 & 5 & 0 & 0 & 0 \\
Ref & 0 & 0 & 0 & 2 & 0 & 0 & 0 \\
Ref & 0 & 0 & 0 & 2 & 0 & 0 & 0 \\
P1 & 2 & 1 & 0 & 0 & 1 & 0 & 0 \\
P1 & 0 & 0 & 0 & 0 & 0 & 0 & 0 \\
P1 & 0 & 5 & 0 & 1 & 0 & 0 \\
P1 & 0 & 0 & 0 & 1 & 0 & 0 \\
\hline
\end{tabular}


Table S6. continued (13 of 13)

\begin{tabular}{cccccc}
\hline Station & Thracioidea juv. & Thyasiraflexuosa & Thyasira juv. & $\begin{array}{c}\text { Upogebia } \\
\text { deltaura }\end{array}$ & Upogebia juv. \\
\hline T1 & 0 & 0 & 0 & 0 & 0 \\
T1 & 1 & 0 & 0 & 1 & 0 \\
P0 & 0 & 0 & 0 & 1 & 0 \\
P0 & 0 & 0 & 0 & 1 & 0 \\
P0 & 1 & 0 & 0 & 0 & 0 \\
P0 & 0 & 0 & 1 & 2 & 1 \\
P0 & 0 & 0 & 0 & 1 & 0 \\
Ref & 0 & 2 & 0 & 2 & 0 \\
Ref & 0 & 0 & 0 & 2 & 0 \\
Ref & 0 & 0 & 0 & 0 & 0 \\
P1 & 0 & 0 & 0 & 4 & 0 \\
P1 & 0 & 0 & 0 & 2 & 0 \\
P1 & 0 & 0 & 0 & 0 & 0 \\
P1 & 0 & 0 & 0 & 1 & 0 \\
\hline
\end{tabular}

